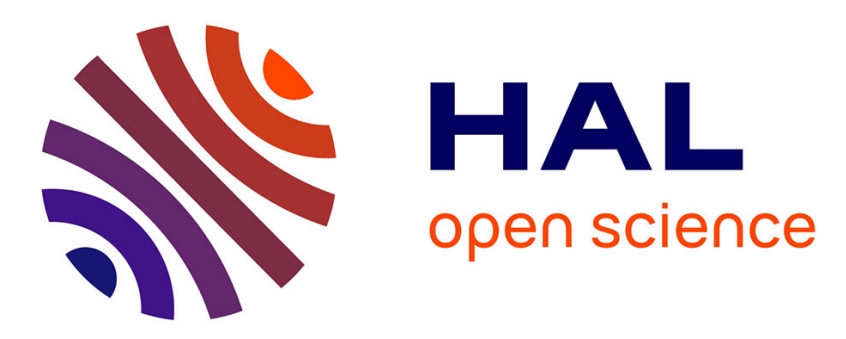

\title{
Wall Slip of Soft-Jammed Systems: A Generic Simple Shear Process
}

\author{
X. Zhang, Elise Lorenceau, Philippe Basset, Tarik Bourouina, Florence \\ Rouyer, Julie Goyon, Philippe Coussot
}

\section{- To cite this version:}

X. Zhang, Elise Lorenceau, Philippe Basset, Tarik Bourouina, Florence Rouyer, et al.. Wall Slip of Soft-Jammed Systems: A Generic Simple Shear Process. Physical Review Letters, 2017, 119 (20), 10.1103/PhysRevLett.119.208004 . hal-01763648

\section{HAL Id: hal-01763648 \\ https://hal.science/hal-01763648}

Submitted on 3 May 2018

HAL is a multi-disciplinary open access archive for the deposit and dissemination of scientific research documents, whether they are published or not. The documents may come from teaching and research institutions in France or abroad, or from public or private research centers.
L'archive ouverte pluridisciplinaire HAL, est destinée au dépôt et à la diffusion de documents scientifiques de niveau recherche, publiés ou non, émanant des établissements d'enseignement et de recherche français ou étrangers, des laboratoires publics ou privés. 


\title{
Wall slip of soft-jammed systems: a generic simple shear process
}

\author{
X. Zhang ${ }^{1}$, E. Lorenceau ${ }^{2}$, P. Basset ${ }^{3}$, T. Bourouina ${ }^{3}$, F. Rouyer ${ }^{1}$, J. Goyon ${ }^{1}$, P. Coussot ${ }^{1}$ \\ ${ }^{1}$ Univ. Paris-Est, Laboratoire Navier (ENPC-IFSTTAR-CNRS), 2 Allée Kepler, 77420 Champs sur Marne, France \\ ${ }^{2}$ Univ. Grenoble-Alpes, CNRS, LIPhy, 38000 Grenoble, France \\ ${ }^{3}$ Univ. Paris-Est, ESYCOM EA 2552, ESIEE Paris-CNAM-UPEM, 5 Bd Descartes, 77420 Champs sur Marne, \\ France
}

\begin{abstract}
From well-controlled long creep tests we show that the residual apparent yield stress observed with soft-jammed systems along smooth surfaces is an artefact due to edge effects. By removing these effects we can determine the stress solely associated with steady state wall slip below the material yield stress. This stress is found to vary linearly with the slip velocity for a wide range of materials whatever the structure, the interaction types between the elements and with the wall, and the concentration. Thus wall slip results from the laminar flow of some given free liquid volume remaining between the (rough) jammed structure formed by the elements, and the smooth wall. This phenomenon may be described by the simple shear flow in a Newtonian liquid layer of uniform thickness. For various systems this equivalent thickness varies in a narrow range $(35 \pm 15 \mathrm{~nm})$.
\end{abstract}

Various materials, such as foams, emulsions, concentrated suspensions and colloids, are soft-jammed systems, i.e. they can flow only when submitted to a stress larger than a yield stress $\left(\tau_{c}\right)$ otherwise they behave as solids [1]. In many situations it is observed that such materials glide along smooth solid surfaces, in the sense that the bulk material apparently moves as a rigid block for a stress lower than $\tau_{c}$ [2-4]. This effect is called wall slip. Its impact on flow characteristics is dramatic since it may make such materials, otherwise at rest, flow at a high velocity under small stresses. Thus wall slip can overturn the standard continuum mechanics description assuming adherence. This modification of the interaction with the walls, can be used to facilitate the transport of products such as in food digestion [5], cosmetic sensory perception [6], coal water slurry in pipes [7], fresh concrete pumping over long-distance [8], removal of food debris and microbial films [9].

Even if it has for a long time been admitted that it results from the formation of a layer with different components than in the bulk [2], the physics of wall slip is still poorly advanced. Note that this effect must not be confused with shear-banding [10] or confinement effects [11] in jammed systems, which occur beyond the yield stress. Here we focus on flow characteristics below $\tau_{c}$, so that such effects are a priori negligible.

To gain quantitative measurement and understanding concerning wall slip, the basic approach consisted to fit a model to the apparent flow curve (shear stress vs velocity) in the slip regime (i.e. for a stress below the yield stress), or directly measure the slip velocity, and possibly discuss the physical origin of the parameters, for the different material classes (concentrated suspensions [12-15], soft particle suspensions [16-17], emulsions [18-20], foams [20-22]). Generally power-law dependencies for the stress vs slip velocity variations were obtained, with an exponent ranging from $1 / 2$ to 1 . An advanced physical explanation [16] assumes that the liquid layer thickness varies with the balance between attractive (due to osmotic pressure resulting from the jammed nature of the system) and repulsive forces (due to lubricating viscous forces). Some similar approaches were developed independently for individual bubbles or bubble film [23] with later further sophistication [24]. In particular, for foams, linear and non-linear regimes were suggested to occur depending on the relative values of colloidal interaction and viscous effects [25]. On the other side, for hard-sphere colloidal suspensions, it was shown [13-15] that a linear velocity variation plus a constant (residual) yield stress term well represents their data, but the liquid thickness associated to the linear term was shown to be independent of the osmotic pressure. Such a residual yield stress was observed in most studies, but it appears somewhat contradictory with the existence of a liquid layer allowing wall slip, and detailed analysis of wall-elements interactions could not identify a clear origin for this effect $[13,18]$. 
Here, by determining precisely the stress vs velocity law from sufficiently long creep tests, we can show that the residual yield stress is due to edge effects (evaporation along the line of contact) and, after its removal, a linear stress vs velocity law is found whatever the type of interactions between the suspended elements, their concentration, and the interactions with the solid surface.

A simple observation provides a straightforward view and analysis of the main characteristics of wall slip: a small volume of a yield stress fluid (an oil-in-water emulsion) placed over an inclined smooth surface slowly moves downwards as a rigid block (see Fig.1a). A detailed observation of the traces left on the surface reveals i) apparent thick tracks of material along the edges of the sample which persists over time, ii) a transient thin liquid layer which soon fully evaporates behind the sample, suggesting that the material is sheared only in an interstitial liquid layer along the wall.
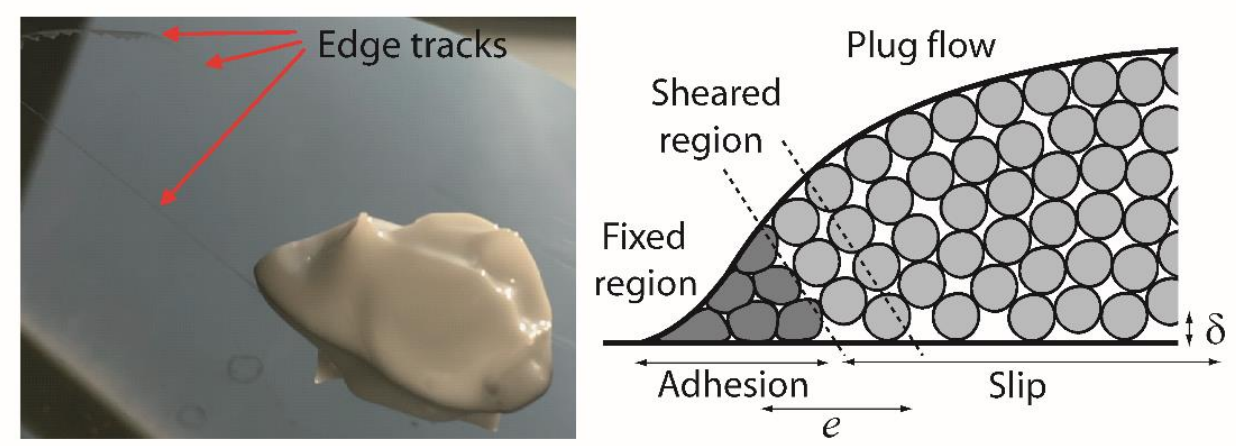

Figure 1: (a) View of a heap of emulsion after some motion (from the top left) on a smooth inclined surface. (b) Schematic aspect of the sample cross-section close to the contact line showing the different flow regions and their microstructural origin (suspended elements in dark or light grey, interstitial liquid in white).

In order to get a precise information on wall slip of such material we follow a procedure which allows to clearly identify the rheological characteristics of simple or thixotropic [26] yield stress fluids. This consists to apply a shear stress $(\tau)$ to the material and follow its apparent deformation $(\gamma)$ in time $(t)$. Here we use a rheometer equipped with parallel plates with one rough surface and one smooth surface (i.e. of roughness much smaller than the element size, see [27]), separated by a distance (i.e. gap) $h . \tau$ and $\gamma$ are computed from the torque and rotation velocity, through expressions corresponding approximately to the local values at $3 / 4$ of the distance from the axis for a Newtonian or a yield stress fluid [27]. The test is then repeated at another stress level, with the material prepared in the same initial state (preshear then short rest). Under such conditions the overall results are reproducible and do not depend on the order of the tests at different stress values.

First we focus on a concentrated emulsion (see [27]), in which the element interactions are essentially repulsive. We observe two distinct regimes (see inset of Fig.2): for $\tau$ smaller than a critical value $\left(\tau_{c}{ }^{\prime}\right)$ the material is just slightly deformed over a short time, then $\gamma$ reaches a plateau indicating that no more motion occurs; for stresses larger than $\tau_{c}{ }^{\prime}$, after a short period $\gamma$ increases linearly with time, indicating that the material flows steadily. In that case we can define $\dot{\gamma}$, the apparent steady state shear rate associated to $\tau$, as the slope of the $\gamma(t)$ curve. The same experiment with two rough surfaces provides two similar regimes, but with a transition now observed at a critical stress equal to $\tau_{c}$, the yield stress of the material above which the material flows in bulk, with $\tau_{c}{ }^{\prime}<\tau_{c}$. We deduce that for $\tau_{c}{ }^{\prime}<\tau<\tau_{c}$, the material may flow only in a thin (slip) layer along the smooth wall, in agreement with the observations in Figure 1 . This is confirmed by the observation that a vertical mark at the sample periphery moves as a rigid 
block attached with the rough plate. Moreover, tests with different gaps for the same stress give an apparent shear rate increasing with the inverse of the gap, which means that the flow characteristics in this layer only depend on $\tau$. We can then define the slip velocity $\left(V_{S}=\dot{i h}\right)$ as the relative velocity between this block and the smooth wall.

It is now possible to represent the apparent flow curve of the material, i.e. $\tau$ vs $\dot{\gamma}$, which appears to be made of two parts (see Fig.2): below $\tau_{c}, \tau$ strongly increases with $\dot{\gamma}$; beyond $\tau_{c}$, the curve flattens and soon joins the effective flow curve of the bulk material (obtained with rough surfaces), indicating that slip can still occur but becomes negligible with regards to the flow rate induced by bulk flow, when $\tau$ increases. We thus get the standard aspect of apparent flow curves with smooth surfaces, as observed with a variety of simple yield stress fluids for which, as for our materials, repulsive interactions between suspended elements dominate [12-15, 18-22].

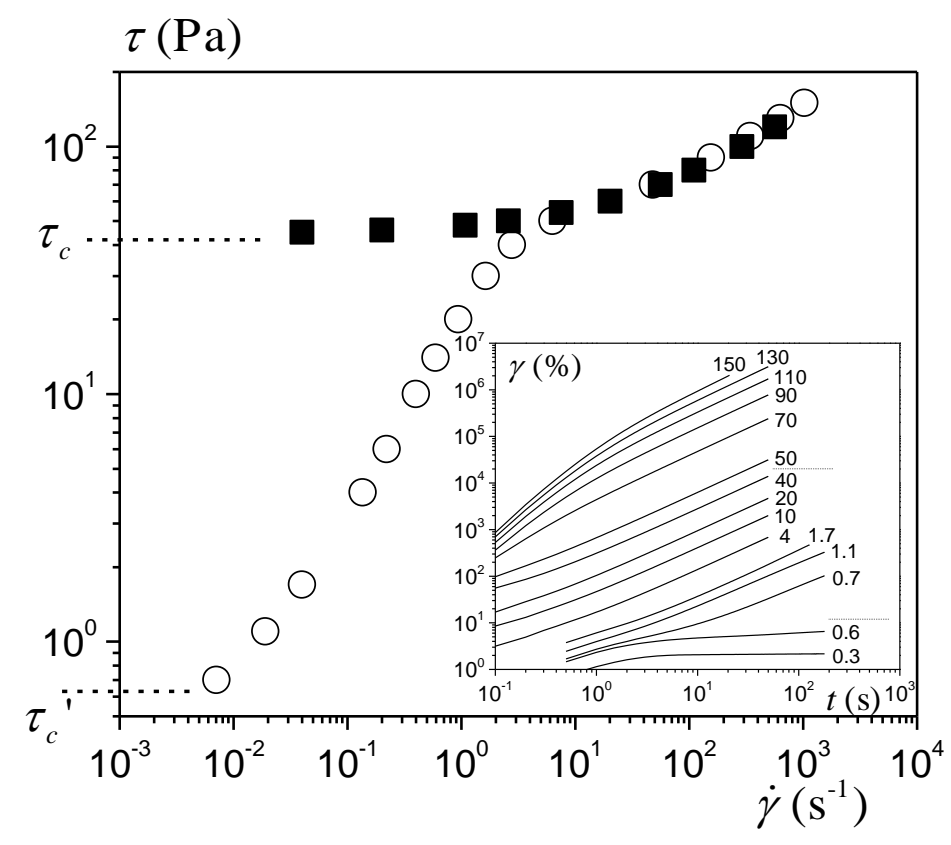

Figure 2: Direct concentrated emulsion (82\%): flow curve (with rough surfaces) (filled squares) and apparent flow curve in the presence of wall slip along one smooth surface (open circles). The inset shows creep test data for the latter case. The numbers correspond to stress values in Pascals. The dotted lines mark the transition to slip (lower line) and the bulk yielding (upper line).

Let us now focus on materials in which suspended element interactions are essentially attractive (i.e. weak flocculation) (see [27]). In general such materials exhibit significant thixotropy, due to their relatively long time of restructuring. Our rheometrical protocol with rough surfaces allows to get the typical flow curve [10] similar to that of a simple yield stress fluid, except that no (homogeneous) steady-state flow may be obtained below a critical shear rate $\dot{\gamma}_{c}$ (see Fig.3). During creep tests there is a viscosity bifurcation [28]: the material first significantly flows (it undergoes very large deformation) and finally evolves either towards stoppage ( $\dot{\gamma}=0$ ) or to $\dot{\gamma}>\dot{\gamma}_{c}$ around $\tau_{c}$ (see top inset of Fig.3).

For such a material, with a smooth surface, a steady wall slip occurs for $\tau<\tau_{c}$. Increasing $\tau$ leads to increase $V_{s}$ and when $\tau>\tau_{c}$ wall slip tends to become negligible. Remarkably, we expect that if $\dot{\gamma}_{c}$, which depends on the bulk material properties, is larger than the shear rate at the intersection $\left(\dot{\gamma}_{0}\right)$ of the effective (bulk) flow curve and the wall slip flow curve, there will be a range of $\dot{\gamma}$ in which the material cannot flow steadily if the stress is imposed: the material evolves towards bulk solidification with wall slip leading to $\dot{\gamma} \approx \dot{\gamma}_{0}$, if $\tau=\tau_{c}(1-\varepsilon)$ (with $\varepsilon<<1$ ); it flows and undergoes a significant viscosity decrease so that wall slip is apparently negligible, if $\tau=\tau_{c}(1+\varepsilon)$. This prediction is in agreement with the evolution of the experimental creep curves around $\tau_{c}$, here equal to $27.5 \mathrm{~Pa}$ (see bottom inset of Fig.3) so that, finally, 
in the flow curve, there is effectively a jump (by about one decade) between the maximum shear rate for pure slip flow $\left(\dot{\gamma}_{0}\right)$ and the minimum shear rate associating slip and bulk flow $\left(\dot{\gamma}_{c}\right)$ (see Fig.3). If, on the contrary, $\dot{\gamma}_{c}<\dot{\gamma}_{0}$, there is no such jump, as for simple yield stress fluids. Thus despite a strong change of structure characteristics, there is apparently no significant change in the slip characteristics. The only possible difference is situated at the transition from the slip and the shear regime, due to material thixotropy.

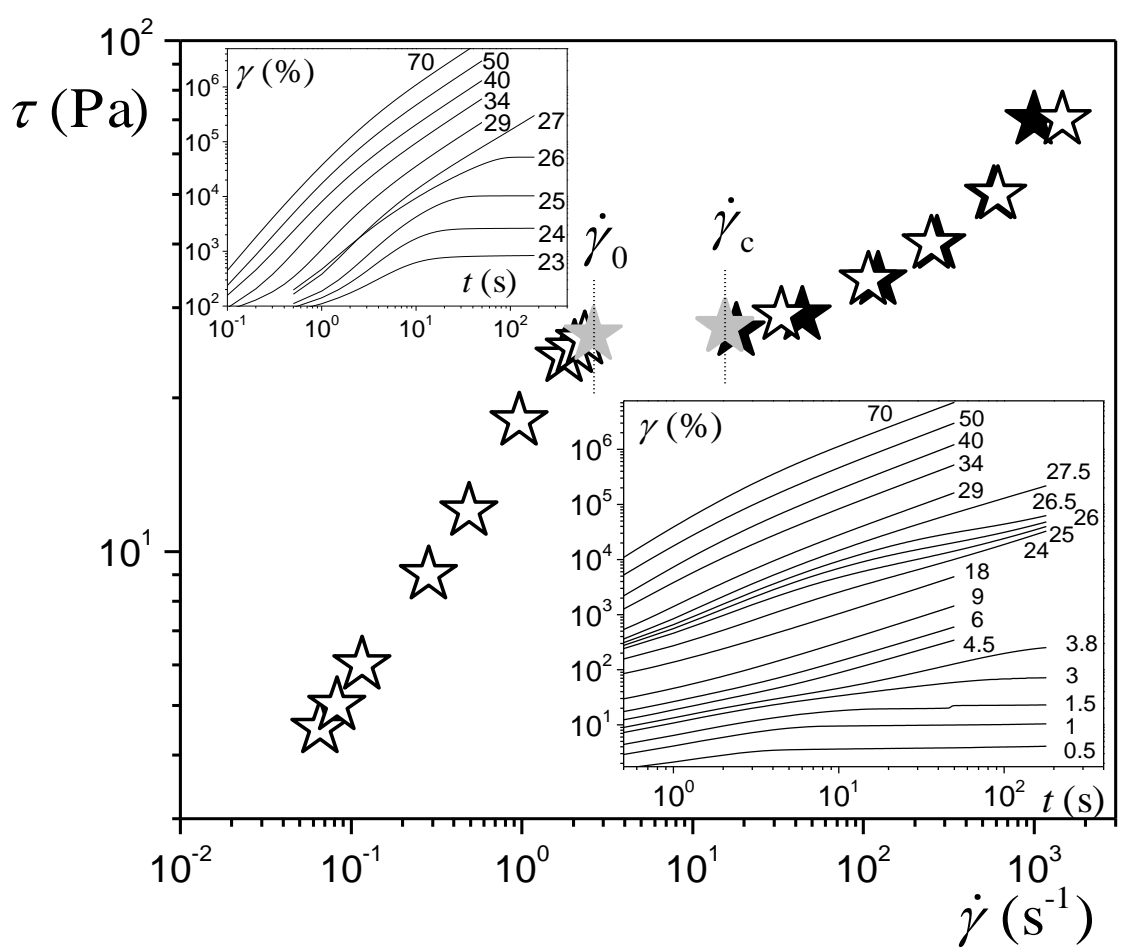

Figure 3: Flow curve a thixotropic clay-water suspension for fully rough (filled stars) and partially smooth (open stars) surface conditions. The insets show creep curves for rough (top) and smooth (bottom) surfaces. The data shown as grey stars (for $27 \mathrm{~Pa}$ ) correspond to the jump in apparent steady state observed for a stress between 24 and $27.5 \mathrm{~Pa}$ (see bottom inset).

With our procedure consisting to wait a sufficient time to reach a clear steady state flow, we can rather precisely identify $\tau_{c}{ }^{\prime}$. For example, for the material of Figure 2 , we can conclude that $\tau_{c}{ }^{\prime}$ is situated between 0.6 and $0.7 \mathrm{~Pa} . \tau_{c}^{\prime}$ has so far been considered as reflecting some attractive interaction between the suspended elements and the solid surface [29]. However, the reproducibility of our measurements in the slip regime, with an uncertainty of the order of $2 \%$ on the apparent flow curve data, is excellent, except at the approach of $\tau_{c}{ }^{\prime}$, where the uncertainty becomes of the order of $20 \%$ (see Fig.4). An even more critical observation is that $\tau_{c}{ }^{\prime}$ significantly increases when the sample diameter is decreased while keeping $h$ constant (see Fig.4). In such a case the flow conditions along most of the wall surface are a priori identical, but if some edge effect induces an additional stress $\left(\tau_{a}\right)$ acting over a constant width $(e)$ along the sample periphery, it will induce an additional component in the apparent stress, proportional to the inverse of the sample radius, i.e. $\tau \approx \tau_{0}+3 e \tau_{a} / R$ (see [27] for detailed analysis). This roughly corresponds to the observed trend (see inset of Fig.4).

Our assumption is supported by the observation of a track stuck on the plane surface along the line of contact of the heap after its displacement (see Fig.1a). This means that there is some flow of the material in a thin (bulk) volume along the contact line (see Fig.1b). A similar flow certainly occurs at the sample periphery in a rheometrical test, which requires to apply a stress larger than $\tau_{c}$ in a thin region located near the periphery, and leads to an apparent stress likely increasing with the local shear rate but tending to $\tau_{c}{ }^{\prime}$ 
at low $\dot{\gamma}$. The origin of this effect might be that some suspended elements tend to be attached to the solid surface at the contact line (see Fig.1b), as a result of a slight evaporation effect at the contact line [30]. This statement is confirmed by a simple test: if we significantly limit the evaporation by coating the sample periphery with a thin oil film just after its set up on the bottom plane, the apparent $\tau_{c}{ }^{\prime}$ effect almost disappears (see Fig.4). The basic ingredients required to see this effect, which are the existence of a contact line, suspended elements and evaporation, are present in most of the experimental situations.

Under these conditions it is crucial to remove the stress associated with edge effects from the apparent stress. As a first approximation this may be done by withdrawing $\tau_{c}{ }^{\prime}$ from $\tau$, thus neglecting some possible slight increase of the edge effect stress with $\dot{\gamma}$. In that case all the data for different sample diameters fall along a master curve (see inset of Fig.4), which means that we have obtained a consistent apparent flow curve in the slip regime whatever the sample dimension, and this flow curve is strictly associated with wall slip effect.

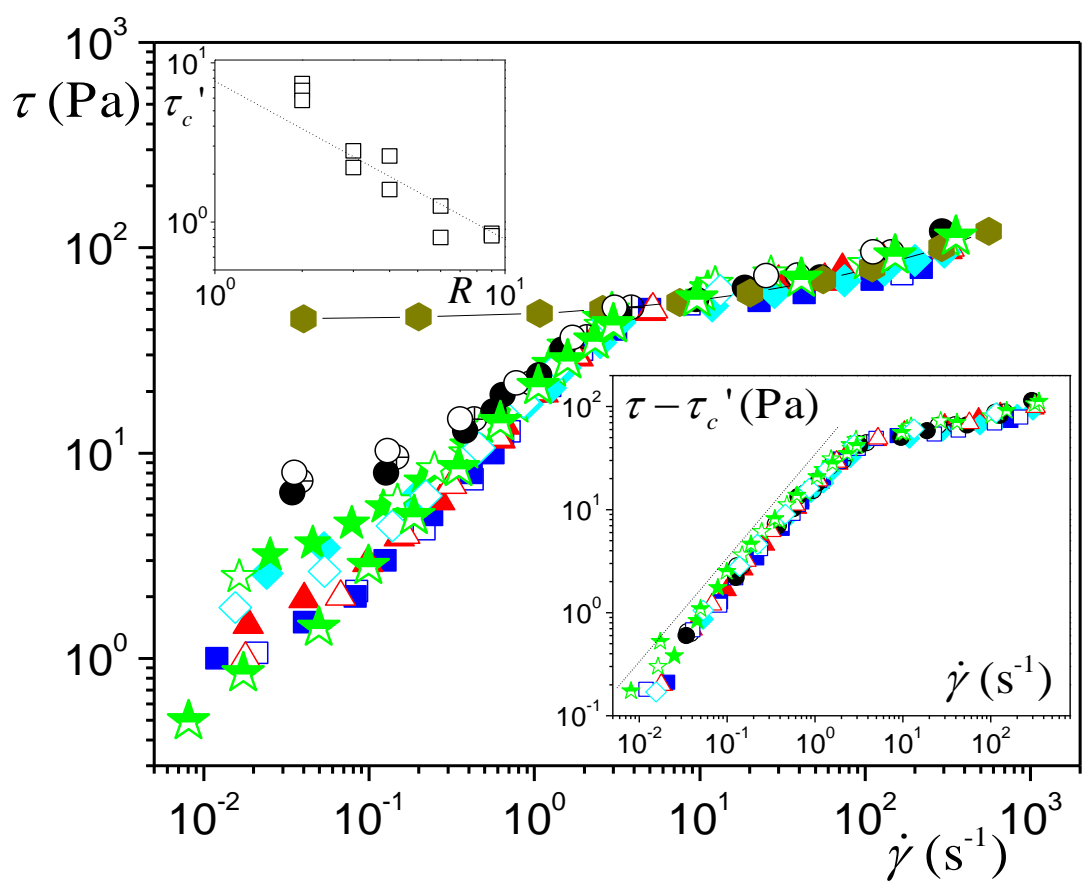

Figure 4: Apparent flow curves of an emulsion (82\%) for same gap and different plate diameters: $9 \mathrm{~cm}$ (squares), $6 \mathrm{~cm}$ (triangles), $4 \mathrm{~cm}$ (diamonds), $3 \mathrm{~cm}$ (stars), $3 \mathrm{~cm}$ with oil film at the sample periphery (half-filled stars), $2 \mathrm{~cm}$ (circles). Hexagons show data with two rough plate surfaces. Several tests carried out by changing the sample while keeping the same surface are shown for each diameter in order to appreciate the reproducibility. Note that in order to remove the impact of sample shape at the periphery which increases when sample $R$ decreases $\tau$ has been rescaled by a factor around 1 to get the same stress values for $\dot{\gamma}_{0}$ (see [24]). The bottom inset shows the stress minus the residual yield stress vs shear rate. The dotted line of slope 1 is a guide for the eye. The top inset shows $\tau_{c}{ }^{\prime}$ (in $\mathrm{Pa}$ ) values as a function of $R$ (in $\mathrm{cm})$.

Therefore, we can focus on these intrinsic wall slip properties by looking at the variation of $V_{S}$ as a function of the shear stress strictly associated with wall slip $\tau_{s}=\tau-\tau_{c}{ }^{\prime}$, i.e. in the absence of edge effects. We find that $V_{S}$ essentially follows a straight line of slope 1, over the whole range of $\tau_{s}$ (see Figs. 4-5). Note that our procedure for the determination of $\tau_{c}{ }^{\prime}$ allows to avoid any significant ambiguity about the observed slope of variation (i.e. 1 ), whereas the use of an arbitrary lower value for $\tau_{c}{ }^{\prime}$ may, particularly when $\tau_{c}{ }^{\prime}$ is not much smaller than $\tau_{c}$, wrongly lead to conclude to $V_{S} \propto \tau_{S}{ }^{p}$ with $p$ distinctly larger than 1 . In order to compare the different data relatively to the regime change observed for $\tau_{S}=\tau_{c}, V_{S}$ and $\tau_{S}$ must be 
rescaled by $\tau_{c}$. This makes it possible to observe (see Fig.5) a general tendency to a slope slightly larger than 1 at the approach of the regime change, say in the range $\left[\tau_{c} / 2 ; \tau_{c}\right]$, which might reflect a tendency to lubricational repulsion as expected from models $[16,18,23]$.

In addition $V_{S}$ is inversely proportional to the interstitial liquid viscosity $(\mu)$ for emulsions with the same concentration and droplet size (see Fig.5). This finally means that a general wall slip law: $\tau_{s}=\mu V_{s} / \delta$ for $\tau<\tau_{c}$, well represents the data in a three-decade range of $\tau_{s} / \tau_{c}$, with $\delta$ a material parameter.

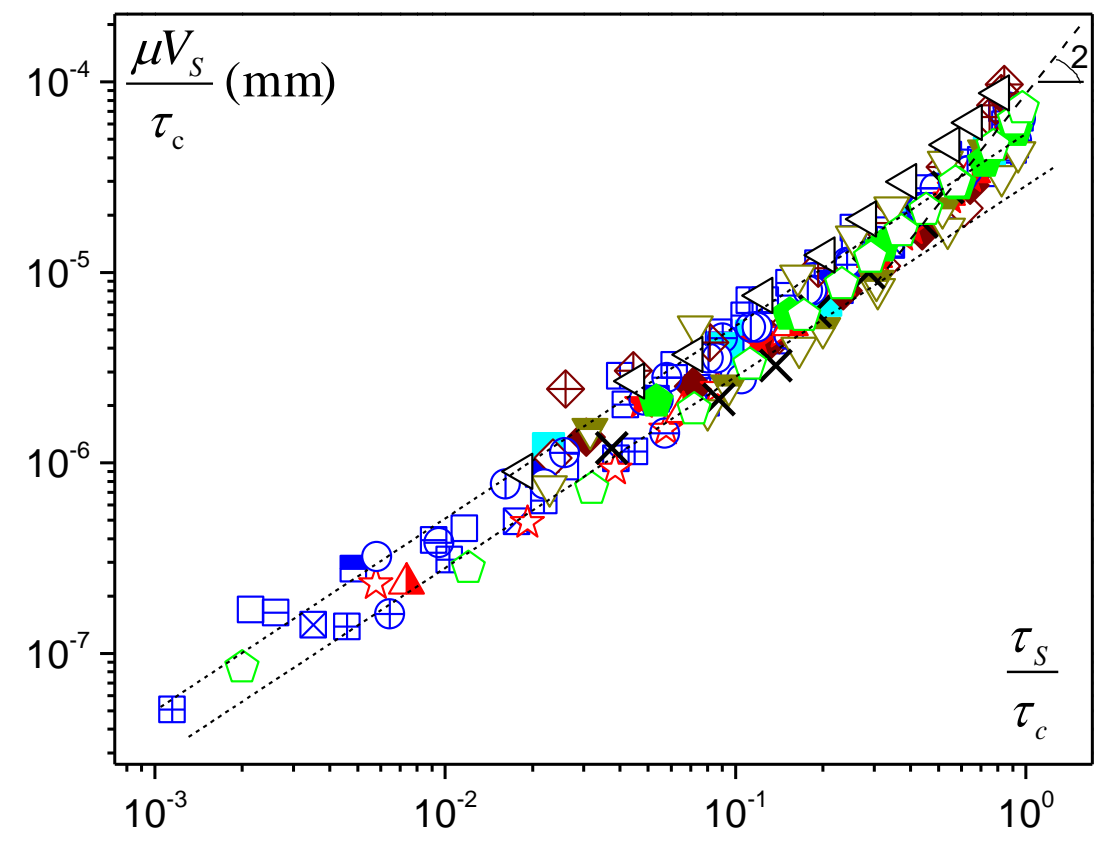

Figure 5: Wall slip velocity for various materials (see [27] for detailed data) as a function of slip stress: emulsions at different concentrations on silicon surface (blue squares), emulsions with different surfactants and solid surfaces (blue circles), emulsion with water-glycerol solution (with viscosity 15 times that of water) as interstitial liquid (light blue filled square), bentonite suspensions at different concentrations (brown diamonds), inverse emulsions (dark yellow inverse triangles) (here using $1.5 \mu$ (see [27]), Carbopol gels (green pentagons), ketchup (red filled stars), mustard (red open stars), foam (crosses), direct emulsion (82\%) over a Teflon surface (black cones). The dashed lines correspond to the equation $\tau_{s}=\mu V_{s} / \delta$ with $\delta=30 \mathrm{~nm}$ and $\delta=50 \mathrm{~nm}$.

Such results are apparently consistent with the existence of a uniform layer of thickness $\delta$ of a Newtonian liquid solution sheared along the wall while the bulk moves as a solid. Remarkably, assuming this solution essentially corresponds to the interstitial liquid, $\delta$ appears to be situated in a narrow range, say 20-50 nm (see Fig.5), whatever the material structure (emulsion made of droplets with repulsive interactions, flocculated clay suspension, foam, materials with more complex structures), the material concentration (for direct and inverse emulsions, and clay suspension), and for various interactions between the interstitial liquid or the suspended elements and the solid surface (different surfactants in emulsions, different wetting properties of the interstitial liquid on the solid surface) [27].

This similar $V_{S}$ vs $\tau_{s}$ variation along with the remarkable persistency of the value of $\delta$, despite the physicochemical differences between all the materials and surfaces we tested, suggest that the origin of the wall slip for this range of systems lies at first order in their common property: a jammed structure filled with a mobile interstitial liquid. More precisely, any of the above jammed systems is made of elements of size typically ranging in [0.1-10 $\mu \mathrm{m}$ ]. The jammed structure they form is rough, with a roughness typically of the order of the element size and, due to its yield stress this structure shape is likely partly maintained at the approach of the smooth wall, i.e. in contrast with an unjammed material the elements do not particularly 
align along the wall, they keep their 3D distribution, precisely because this is a jammed structure (see Fig.1b). The interstitial liquid fills the distance $(d)$ between the wall and the first element surface, and the displacement of the jammed structure as a block induces a flow of the liquid throughout this 3D porous structure. This is a Stokes flow requiring an average stress proportional to velocity and liquid viscosity, via a single characteristic length $\delta$ reflecting this flow through a more or less complex structure roughly characterized by its porosity and pore size distribution. In our case it happens that our samples with very different structure yield similar $\delta$ values. This might be due to the fact that highly squeezed structure (gels, emulsions, microgels) have a large pore size but a low porosity, while dispersed structure (clay) have a small pore size but a large porosity, two effects which more or less balance and could lead to similar resistance to liquid flow and thus similar apparent wall slip thickness. In this context the second regime observed at the approach of $\tau_{c}$ (see Fig.5) might simply be due to a progressively increasing erosion of the rough external jammed network exhibiting local stress resistances lower than $\tau_{c}$.

Acknowledgements: This work has benefited from fruitful discussions with Michel Cloitre, and from a French government grant managed by ANR within the frame of the national program Investments for the Future ANR-11-LABX-022-01 and ANR-10-EQPX-48-01.

\section{References}

[1] A.J. Liu, S.R. Nagel, Nature, 396, 6706 (1998)

[2] H.A. Barnes, J. Non-Newt. Fluid Mech., 56, 221 (1995)

[3] S. Granick, Y. Zhu, H. Lee, Nature Materials, 2, 221 (2003)

[4] M. Cloitre, R.T. Bonnecaze, Rheol. Acta, 56, 283 (2017)

[5] J.R. Stokes, M.W. Boehm, S.K. Baier, Current Opinion Colloid Interface Sci., 18, 349 (2013)

[6] S. Ozkan, T.W. Gillece, L. Senak., D.J. Moore, Int. J. Cosmetic Sci., 34, 193 (2012)

[7] L. Chen, Y. Duan, C. Zhao, L. Yang, Chem. Eng. Proc., 48, 1241 (2009)

[8] T.T. Ngo, E.H. Kadri, R. Bennacer, F. Cussigh, Construction Building Mat., 24, 1253 (2010)

[9] N. Rungraeng, S.H. Yoon, Y. Li, S. Jun, Trans. ASABE, 58, 861 (2015)

[10] G. Ovarlez, S. Rodts, X. Chateau, P. Coussot, Rheol. Acta, 48, 831 (2009)

[11] J. Goyon, A. Colin, L. Bocquet, Soft Matter, 6, 2668 (2010)

[12] U Yilmazer, D.M. Kalyon, J. Rheol., 33, 1197 (1989); H.J. Walls, S.B. Caines, A.M. Sanchez, S.A. Khan, J. Rheol., 47, 847 (2003)

[13] P. Ballesta, R. Besseling, L. Isa, G. Petekidis, W.C.K. Poon, Phys. Rev. Lett., 101, 258301 (2008)

[14] P. Ballesta, G. Petekidis, L. Isa, W.C.K. Poon, R. Besseling, J. Rheol., 56, 1005 (2012)

[15] P. Ballesta, N. Koumakis, R. Besseling, W.C.K. Poon, G. Petekidis, Soft Matter, 9, 3237 (2013)

[16] S.P. Meeker, R.T. Bonnecaze, M. Cloitre, Phys. Rev. Lett., 92, 198302 (2004)

[17] T. Divoux, V. Lapeyre, V. Ravaine, S. Manneville, Phys. Rev. E, 92, 060301 (2015); S. Atkas, D.M. Kalyon, B.M. Marin-Santibanez, J. Perez-Gonzalez, J. Rheol., 58, 513 (2014); J.F. Ortega-Avila, J. Perez-Gonzalez, B.M. Marin-Santibanez, F. Rodriguez-Gonzalez, S. Atkas, M. Malik, D.M. Kalyon, J. Rheol., 60, 503 (2016)

[18] S.P. Meeker, R.T. Bonnecaze, M. Cloitre, J. Rheol., 48, 1295 (2004)

[19] J.B. Salmon, L. Becu, S. Manneville, A. Colin, Eur. Phys. J. E, 10, 209 (2003)

[20] V. Bertola, F. Bertrand, H. Tabuteau, D. Bonn, P. Coussot, J. Rheol., 47, 1211 (2003)

[21] N.D. Denkov, S. Tcholakova, K. Golmeanov, K.A. Ananthpadmanabhan, A. Lips, Soft Matter, 5, 3389 (2009)

[22] S. Marze, D. Langevin, A. Saint-Jalmes, J. Rheol., 52, 1091 (2008)

[23] N.D. Denkov, V. Subramanian, D. Gurovich, A. Lips, Colloids and Surfaces A : Physicochem. Eng. Aspects, 263, 129 (2005) 
[24] N.D. Denkov, S. Tcholakova, K. Golemanov, V. Subramanian, A. Lips, Colloids and Surfaces A :

Physicochem. Eng. Aspects, 282, 329 (2006)

[25] M. Le Merrer, R. Lespiat, R. Höhler, S. Cohen-Addad, Soft Matter, 11, 368 (2015)

[26] P. Coussot, H. Tabuteau, X. Chateau, L. Tocquer, G. Ovarlez, J. Rheol., 50, 975 (2006)

[27] Supplementary material : Materials - Surface characteristics - Rheometry - Detailed results for the different materials

[28] P. Coussot, Q.D. Nguyen, H.T. Huynh, D. Bonn, Physical Review Letters, 88, 175501 (2002); P. Coussot, Q.D. Nguyen, H.T. Huynh, D. Bonn, J. Rheol., 46, 573 (2002)

[29] J.R. Seth, M. Cloitre, R.T. Bonnecaze, J. Rheol., 52, 1241 (2008)

[30] R.D. Deegan, O. Bakajin, F. Dupont, G. Huber, S.R. Nagel, T.A. Witten, Nature, 389, 827 (1997)

[31] H. Van Damme, P. Levitz, J.J. Fripiat, J.F. Alcover, L. Gatineau, F. Bergaya, pp. 24-30 in Physics of Finely Divided Matter (N. Boccara and M. Daoud, Eds., Springer Verlag, Berlin, 1985)

[32] M. Morvan, D. Espinat, J. Lambard, T. Zemb, Colloids Surfaces A : Physicochem. Eng. Aspects, 82, 193 (1994)

[33] J.M. Piau, J. Non-Newt. Fluid Mech., 144, 1 (2007)

[34] J. Boujlel, M. Maillard, A. Lindner, G. Ovarlez, X. Chateau, P. Coussot, J. Rheol., 56, 1083-1108 (2012) 